Laurence Orchard*, Matthew Baldry, Myra Nasim-Mohi, Chantelle Monck, Kordo Saeed, Michael P. W. Grocott and Dushianthan Ahilanandan on behalf of the University Hospital Southampton Critical Care Team and the REACT COVID Investigators

\title{
Vitamin-D levels and intensive care unit outcomes of a cohort of critically ill COVID-19 patients
}

https://doi.org/10.1515/cclm-2020-1567

Received October 21, 2020; accepted December 28, 2020;

published online January 19, 2021

\section{Abstract}

Objectives: The pattern of global COVID-19 has caused many to propose a possible link between susceptibility, severity and vitamin-D levels. Vitamin-D has known

UHS Critical Care Clinical Team: Dr. Sanjay Gupta, Dr. Julian Nixon, Professor Michael P W Grocott, Professor Denny ZH Levett, Dr. Michael Stewart, Dr. Ahilanadan Dushianthan, Dr. David Sparkes, Dr. Robert Chambers, Dr. Kathleen Nolan, Dr. Suzie Tanser, Dr. Jonathan Fennell, Dr. Michael Celinski, Dr. Dominic Richardson, Dr. Rebecca Cusack, Dr. Benjamin Skinner, Dr. Timothy Nicholson-Robert, Dr. Mai Wakatsuki, Dr. Ben Thomas, Dr. Francois Wessels.

REACT Investigators: Professor Tom Wilkinson, Dr. Anna Freeman, Dr. Hannah Burke, Dr. Ahilanadan Dushianthan, Dr. Michael Celinski, Professor James Batchelor, Professor Saul Faust, Professor Gareth Thomas, Professor Christopher Kip.

*Corresponding author: Dr. Laurence Orchard, General Intensive Care Unit, University Hospital Southampton NHS Foundation Trust,

Tremona Road, Southampton, UK, Phone: +44 7791190726,

E-mail: laurence.orchard@nhs.net

Matthew Baldry and Myra Nasim-Mohi, General Intensive Care Unit, University Hospital Southampton NHS Foundation Trust,

Southampton, UK

Chantelle Monck, Southampton Specialist Virology Centre, University of Southampton School of Medicine, University Hospital Southampton NHS Foundation Trust, Southampton, UK

Kordo Saeed, Microbiology Innovation and Research Unit, Department of Microbiology, University Hospital Southampton NHS Foundation Trust, Southampton, UK; Faculty of Medicine, University of Southampton University, Hospital Southampton, Southampton, UK Michael P. W. Grocott and Dushianthan Ahilanandan, General Intensive Care Unit, University Hospital Southampton NHS Foundation Trust, Southampton, UK; Faculty of Medicine, University of Southampton University, Hospital Southampton, Southampton, UK; and NIHR Southampton Clinical Research Facility and NIHR Southampton Biomedical Research Centre, University Hospital Southampton/University of Southampton, Southampton, UK immune modulatory effects and deficiency has been linked to increased severity of viral infections.

Methods: We evaluated patients admitted with confirmed SARS-COV-2 to our hospital between March-June 2020. Demographics and outcomes were assessed for those admitted to the intensive care unit (ICU) with normal $(>50 \mathrm{nmol} / \mathrm{L})$ and low $(<50 \mathrm{nmol} / \mathrm{L})$ vitamin-D.

Results: There were 646 SARS-COV-2 PCR positive hospitalisations and 165 (25.5\%) had plasma vitamin-D levels. Fifty patients were admitted to ICU. There was no difference in vitamin-D levels of those hospitalised (34, IQR 18.5-66 nmol/L) and those admitted to the ICU (31.5, IQR 21-42 nmol/L). Higher proportion of vitamin-D deficiency $(<50 \mathrm{nmol} / \mathrm{L})$ noted in the ICU group (82.0 vs. 65.2\%). Among the ICU patients, low vitamin D level $(<50 \mathrm{nmol} / \mathrm{L})$ was associated with younger age ( $57 \mathrm{vs.} 67$ years, $\mathrm{p}=0.04$ ) and lower cycle threshold (CT) real time polymerase chain reaction values (RT-PCR) (26.96 vs. 33.6, $\mathrm{p}=0.02$ ) analogous to higher viral loads. However, there were no significant differences in ICU clinical outcomes (invasive and non-invasive mechanical ventilation, acute kidney injury and mechanical ventilation and hospital days) between patients with low and normal vitamin-D levels.

Conclusions: Despite the association of low vitamin-D levels with low CT values, there is no difference in clinical outcomes in this small cohort of critically ill COVID-19 patients. The complex relationship between vitamin-D levels and COVID-19 infection needs further exploration with large scale randomized controlled trials.

Keywords: COVID-19; intensive care; vitamin-D.

\section{Introduction}

The ongoing COVID-19 (coronavirus disease-19) pandemic remains at the forefront of global biomedical research efforts. The causative enveloped RNA virus, SARS-CoV-2 (severe acute respiratory syndrome coronavirus-2) is highly contagious through respiratory droplets, and so far, the current pandemic is linked to worldwide mortality of more 
than a million. Unfortunately, there are limitations in the understanding of the exact pathophysiological process that leads to the development of critical illness and hypoxic respiratory failure, which occurs in a minority of patients with COVID-19 infection [1].

Although several targeted therapies are currently being evaluated, few have been found to be effective and corticosteroids appear to improve survival in a select group of patients requiring oxygen therapy [2]. Several risk factors, such as older age, ethnicity, raised body mass index (BMI), the presence of diabetes mellitus, and cardiovascular disease, are postulated to be associated with increased risk of infection and subsequent progression to critical illness $[1,3]$. Moreover, nutritional imbalance, including vitamin-D deficiency, has been proposed to increase the rate and severity of the COVID-19 infection [4, 5].

Vitamin-D is a fat-soluble hormone, of which deficiency has been linked to increased risk of COVID-19 infection and severity [6, 7]. An association between viral infections and vitamin-D deficiency has been long recognised, particularly in winter months where the vitamin-D levels are in general at their lowest $[8,9]$. During the first wave of the pandemic in the UK, the National Institute for Health and Care Excellence (NICE) published a rapid review of the current literature relating to vitamin-D treatment for COVID-19 and concluded that there was "no evidence to support vitamin-D supplements to prevent or treat COVID-19' [10]. However, since then, there have been several observational cohort studies suggesting an association between low vitamin-D levels and worse clinical outcomes. To explore this further, we evaluated the vitamin-D levels in a cohort of critically ill COVID-19 patients to review any associations between severity of illness and outcomes.

\section{Materials and methods}

This is a cohort study of COVID-19 patients admitted to the University Hospital Southampton and that subsequently required organ supportive measures in the General Intensive Care Unit. All patients included in the cohort had a positive test for SARS-CoV-2 following a real time polymerase chain reaction (RT-PCR) test from nose and throat swabs, or from tracheal secretions obtained after endotracheal intubation. Samples were transported in VIROCULT virus transport medium. Samples were extracted and purified using magnetic particle extraction on the Thermo Scientific KingFisher Flex. PCR amplification was performed on the Applied Biosystems (ABI) 7,500 by using the Viasure SAR-CoV-2 RT-PCR kit, targeting ORF1ab and $N$ gene. Additionally, primers and probes for the World Health Organization $E$ gene assay (including an internal positive amplification control from extraction), were also used to enhance the sensitivity.
Positive PCR test results were recorded between 19th of March and 15th of June 2020 and the clinical outcomes are up to date as of 30th of September 2020. Due to the observational nature of the study, only patients who had serum vitamin-D levels measured during this admission are included in the study. This study was part of a larger observational cohort study (REACT-COVID Observational Database) and has the approval of the local research and development and National Heath Research Authority (IRAS:285145) with a REC Reference 20/HRA/2986.

Vitamin-D is measured by DxI800 (Beckman Coulter, Brea, CA, USA). The total coefficient of variations (CV) provided by the manufacturer were $9.3 \%$ for $38.94 \mathrm{nmol} / \mathrm{L}$ and $5.6 \%$ for $399 \mathrm{nmol} / \mathrm{L}$. Linearity was given as 4.99-524 nmol/L. All hospitalised COVID-19 patients with admission vitamin-D levels were included in the study. We defined a low serum vitamin-D level as less than $50 \mathrm{nmol} / \mathrm{L}$ in accordance with the local hospital laboratory standards. Detailed analysis was performed for all patients subsequently admitted to the Intensive Care Unit (ICU). Additional data of baseline patient's demographics, such as age, gender, ethnicity, duration of onset, presence of comorbidities, intensive care severity illness scores (Acute Physiology and Chronic Health evaluation-II (APACHE II) and Sequential Organ Failure Assessment (SOFA)), oxygenation severity variables $\left(\mathrm{PaO}_{2} / \mathrm{FiO}_{2}\right)$, admission cycle threshold polymerase chain reaction (CT PCR) values and blood laboratory profiles, were collected from all available hospital electronic databases. The reported clinical outcomes are: the need for mechanical ventilation either invasive or noninvasive; the development of acute kidney injury (AKI) of any stage in accordance with the Kidney Disease Improving Global Outcome (KDIGO) criteria [11]; and the duration of intensive care. Hospital length of stay and overall hospital mortality were also documented.

The continuous variables are expressed as median and interquartile range. Nominal variables are summarised as numbers and percentages. For comparisons between patients with normal ( $>50 \mathrm{nmol} / \mathrm{L})$ and low (<50 nmol/L) vitamin-D levels, Fisher's exact test and Mann-Whitney U test were performed. Pearson correlation coefficient ( $\mathrm{r}$ ) was used to assess the correlation between various variables, including ICU severity scores (APACHE-II and SOFA) and degree of hypoxia on admission. All statistical analyses were caried out using MedCalc (Version 19.4.0) and Graph Pad Prism (Version 8.0.0).

\section{Results}

There were 646 SARS-CoV-2 positive patients hospitalised during this period. Among those, 165 patients (25.5\%) had a plasma vitamin-D level measured during admission. The local hospital standardised cut off value for the plasma vitamin-D level was $50 \mathrm{nmol} / \mathrm{L}$. Forty-nine patients (29.7\%) had normal levels $(>50 \mathrm{nmol} / \mathrm{L})$ and the rest $(70.3 \%)$ had vitamin-D deficiency ( $<50 \mathrm{nmol} / \mathrm{L})$ as defined by the local laboratory standards. Among those who had vitamin-D measurements, 50 patients were admitted to the ICU, and of those, $82 \%$ had levels less than $50 \mathrm{nmol} / \mathrm{L}$ (Figure 1). For those admitted to the ICU and with vitamin-D 


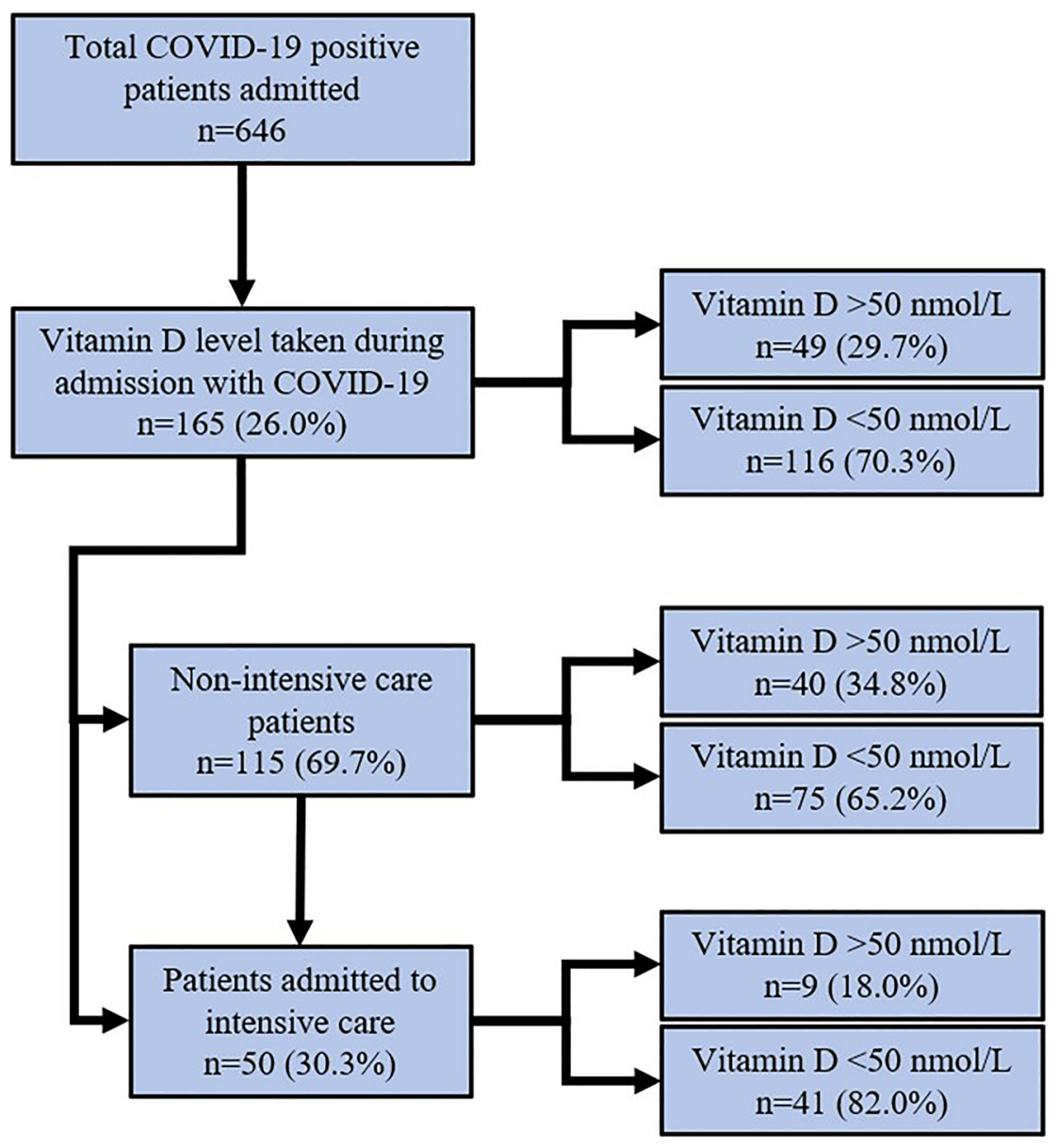

Figure 1: Flow chart displaying the proportion of patients who were admitted with COVID-19, had a vitamin-D level taken during admission and required ITU admission. As well as the proportions within each group with normal ( $>50 \mathrm{nmol} / \mathrm{L})$ and low ( $<50 \mathrm{nmol} / \mathrm{L})$ vitamin-D levels. measurements $(n=50)$, the median age was 60 (IQR 51-67) and the majority needed respiratory support either in the form of invasive $(72 \%)$ or non-invasive $(13 \%)$ mechanical ventilation. The baseline demographics, ethnicity, comorbidities, and the laboratory variables at ICU admission are presented in Table 1. For comparison, the demographics of the hospitalised non-ICU patients are presented in Supplementary Material (Table 1). The ethnicity is differentiated and presented as five groups (White, Asian/Indian, Black, other/mixed, and unknown). Details of ethnicity from the studied group is presented in Supplementary Material (Table 2).

The ICU admission demographics and baseline variables between patients with normal and low vitamin-D levels showed similar baseline characteristics, except for age, presence of hypertension and other comorbidities, and a worse admission $\mathrm{PaO}_{2} / \mathrm{FiO}_{2}$ ratio. It appears patients with normal vitamin-D levels were older and had more severe hypoxemia on admission. The median vitamin-D levels for hospitalised and ICU patients were $34 \mathrm{nmol} / \mathrm{L}$ (IQR 18.5-66.0) and $31.5 \mathrm{nmol} /$ L (IQR 21.0-42.0) $(\mathrm{p}=0.41)$ respectively (Figure 2A).
Among the ICU patients there was no difference in the vitamin-D levels between White Caucasians and the rest of the ethnic groups (Figure 2B). However, ethnicity other than White Caucasians was associated with increased incidence of low vitamin-D levels; $94.4 \%$ of the ethnic groups consisting of Black and Asians had levels $<50 \mathrm{nmol} / \mathrm{L}$, compared to $74.2 \%$ of White Caucasians. The ranges of vitamin-D levels measured varied between patients and $8 \%$ had very low levels of $<10 \mathrm{nmol} / \mathrm{L}$ (Figure 2C).

The ICU group with low vitamin-D levels (<50 nmol/L) had a significantly lower mean cycle threshold (CT) for real time polymerize chain reaction (PCR) value: 27.0 for the low vitamin-D ( $<50 \mathrm{nmol} / \mathrm{L})$ group vs. 33.6 for the group with normal vitamin-D levels ( $>50 \mathrm{nmol} / \mathrm{L}) \quad \mathrm{p}=0.02$ (Figure 2D and Table 1).

We assessed ICU admission disease severity indices alongside plasma vitamin-D levels by using scatter plots with further subgrouping according to the ethnicity. We subsequently found no associations with ICU severity indices and plasma vitamin-D levels (Figure 3). The overall ICU outcomes of these patients are presented in Table 2. 
Table 1: All COVID-19 patients admitted to ICU demographics on admission, disease severity indices, intensive care interventions and admission laboratory markers.

\begin{tabular}{|c|c|c|c|c|}
\hline Demographics ICU & Tested patients, $n=50$ & Vitamin-D levels $>50, n=9$ & Vitamin-D levels $<50, n=41$ & p-Value \\
\hline Age, years & $60.0(51.2-67.0)$ & $67.0(62.0-72.0)$ & $57.0(49.0-66.0)$ & $0.04^{\mathrm{a}}$ \\
\hline Male, n (\%) & $28(56.0 \%)$ & $4(44.0 \%)$ & $24(58.0 \%)$ & 1.00 \\
\hline Symptomatic days prior to hospitalisation & $7.0(5.0-10.0)$ & $7.0(7.0-10.0)$ & $7(5.0-10.0)$ & 0.97 \\
\hline $\mathrm{BMI}, \mathrm{kg} / \mathrm{m}^{2}$ & $27.5(22.8-32.7)$ & $31.0(20.8-33.6)$ & $27.3(23.2-32.5)$ & 0.84 \\
\hline \multicolumn{5}{|l|}{ Race/ethnic group } \\
\hline White & $31(62.0 \%)$ & $8(89.0 \%)$ & $23(56.0 \%)$ & 0.12 \\
\hline Black & $5(10.0 \%)$ & $0(0.0 \%)$ & $5(12.0 \%)$ & 0.56 \\
\hline Asian/Indian & $12(24.0 \%)$ & $1(11.0 \%)$ & $11(27.0 \%)$ & 0.42 \\
\hline Mixed/other & $1(2.0 \%)$ & $0(0.0 \%)$ & $1(2.0 \%)$ & 1.00 \\
\hline Unknown & $1(2.0 \%)$ & $0(0.0 \%)$ & $1(2.0 \%)$ & 1.00 \\
\hline \multicolumn{5}{|l|}{ Comorbidities, n (\%) } \\
\hline Type I diabetes mellitus & $1(2.0 \%)$ & $0(0.0 \%)$ & $1(2.4 \%)$ & 1.00 \\
\hline Type II diabetes mellitus & $14(28.0 \%)$ & $3(33.3 \%)$ & $11(26.8 \%)$ & 0.69 \\
\hline Asthma & 5 (10.0\%) & $2(22.2 \%)$ & $3(7.3 \%)$ & 0.21 \\
\hline COPD & $2(4.0 \%)$ & $0(0.0 \%)$ & $2(4.9 \%)$ & 1.00 \\
\hline Other chronic respiratory disorders & $2(4.0 \%)$ & $0(0.0 \%)$ & $2(4.9 \%)$ & 1.00 \\
\hline Hypertension & $20(40.0 \%)$ & $7(77.8 \%)$ & $13(31.7 \%)$ & $0.02^{\mathrm{a}}$ \\
\hline Ischaemic heart disease & $3(6.0 \%)$ & $0(0.0 \%)$ & $3(7.3 \%)$ & 1.00 \\
\hline Congestive cardiac failure & $1(2.0 \%)$ & $0(0.0 \%)$ & $1(2.4 \%)$ & 1.00 \\
\hline Arrhythmia & $1(2.0 \%)$ & $0(0.0 \%)$ & $1(2.4 \%)$ & 1.00 \\
\hline Chronic kidney disease & $2(4.0 \%)$ & $0(0.0 \%)$ & $2(4.9 \%)$ & 1.00 \\
\hline Immunosuppression & $10(20.0 \%)$ & $2(22.2 \%)$ & $8(19.5 \%)$ & 1.00 \\
\hline Corticosteroid use & $1(2.0 \%)$ & $1(11.1 \%)$ & $0(0.0 \%)$ & 0.18 \\
\hline Use of ACEi or ARB & $15(30.0 \%)$ & $5(55.6 \%)$ & $10(24.4 \%)$ & 0.10 \\
\hline Another comorbidity & $25(50.0 \%)$ & $8(88.9 \%)$ & $17(41.5 \%)$ & $0.02^{\mathrm{a}}$ \\
\hline \multicolumn{5}{|l|}{ Severity indices } \\
\hline APACHE II score & $14.5(12.0-24.0)$ & $12.0(10.0-19.0)$ & $15.0(12.0-25.0)$ & 0.84 \\
\hline SOFA score & $4(3.0-6.0)$ & $4.0(3.0-6.0)$ & $4(3.0-7.0)$ & 0.76 \\
\hline $\mathrm{PaO}_{2} / \mathrm{FiO}_{2}$ ratio, $\mathrm{kPa}$ & $14.9(12.9-18.6)$ & $13.0(10.3-14.7)$ & $15.3(13.8-19.6)$ & $0.04^{\mathrm{a}}$ \\
\hline \multicolumn{5}{|l|}{ Admission laboratory profile } \\
\hline Bilirubin, $\mathrm{mmol} / \mathrm{L}$ & $11.5(9.0-17.8)$ & $9.0(8.0-18.0)$ & $12.0(9.0-17.0)$ & 0.36 \\
\hline Creatinine, $\mathrm{mmol} / \mathrm{L}$ & $67.0(51.8-99.8)$ & $62.0(56.0-69.0)$ & $69.0(51.0-101.0)$ & 0.49 \\
\hline Creatine kinase, $\mathrm{U} / \mathrm{L}$ & $137.0(75.8-270.8)$ & $162.0(58.0-278.0)$ & $128.0(84.3-270.8)$ & 0.88 \\
\hline C-reactive protein, $\mathrm{mg} / \mathrm{L}$ & $165.0(118.5-233.8)$ & $147.0(116.0-242.0)$ & $167.0(120.0-221.0)$ & 0.65 \\
\hline Viral CT-PCR & $28.2(22.6-31.3)$ & $33.6(28.6-34.7)$ & $27.0(21.8-30.3)$ & $0.02^{\mathrm{a}}$ \\
\hline D-dimer, mg/L & $631.0(400.0-2043.5)$ & $766.0(613.0-1883.0)$ & $560.0(370.5-1736.0)$ & 0.61 \\
\hline Ferritin, $\mathrm{mg} / \mathrm{L}$ & $928.0(557.5-1,648.8)$ & $758.0(544.5-923.0)$ & $1,002.0(557.5-2034.0)$ & 0.21 \\
\hline Lactate, $\mathrm{mmol} / \mathrm{L}$ & $1.1(0.8-1.3)$ & $1.3(0.8-1.9)$ & $1.1(0.9-1.2)$ & 0.38 \\
\hline $\mathrm{LDH}, \mathrm{U} / \mathrm{L}$ & $1,109(816.0-1,314.0)$ & $719.0(674.0-1,018.5)$ & $1,143(886.5-1,332.0)$ & 0.06 \\
\hline Lymphocytes, $\times 10^{9} / \mathrm{L}$ & $0.8(0.6-1.0)$ & $0.8 .0(0.5-1.0)$ & $0.8(0.6-1.0)$ & 0.76 \\
\hline HS troponin, ng/L & $13.5(8.3-94.5)$ & $11.0(10.0-29.5)$ & $14.0(8.0-103.5)$ & 0.86 \\
\hline White cell counts, $\times 10^{9} / \mathrm{L}$ & $8.7(6.1-13.1)$ & $7.9(7.1-11.7)$ & $8.9(6.0-13.7)$ & 0.76 \\
\hline Vitamin-D, nmol/L & $31.5(21.0-42.0)$ & $65.0(63.0-75.0)$ & $29.0(19.0-35.0)$ & $<0.01^{\mathrm{a}}$ \\
\hline
\end{tabular}

Data are presented as median (interquartile range) or numbers (percentage) unless otherwise stated. ${ }^{\mathrm{a}} \mathrm{p}<0.05$ as assessed by Fisher's exact test for categorical variables and Mann-Whitney U test for continuous variables. ACEi, angiotensin converting enzyme inhibitor; APACHE II, acute physiology and chronic health evaluation II score; ARB, angiotensin receptor blocker; BMI, body mass index; LDH, lactate dehydrogenase; HS troponin, high sensitivity troponin; $\mathrm{PaO}_{2} / \mathrm{FiO}_{2}$, ratio of arterial oxygen partial pressure to fractional inspired oxygen-worst during admission; SOFA, sequential organ failure assessment score; CT-PCR, viral cycle threshold polymerize chain reaction.

The hospital mortality for those with measured vitamin-D levels in ICU was $14 \%$, with no significant difference between patients with normal vitamin-D levels (11\%) and those with low levels (14.6\%). There were no differences in incidence of mechanical ventilation, acute kidney injury and length of hospitalisation between both groups (Table 2 and Figure 4). 

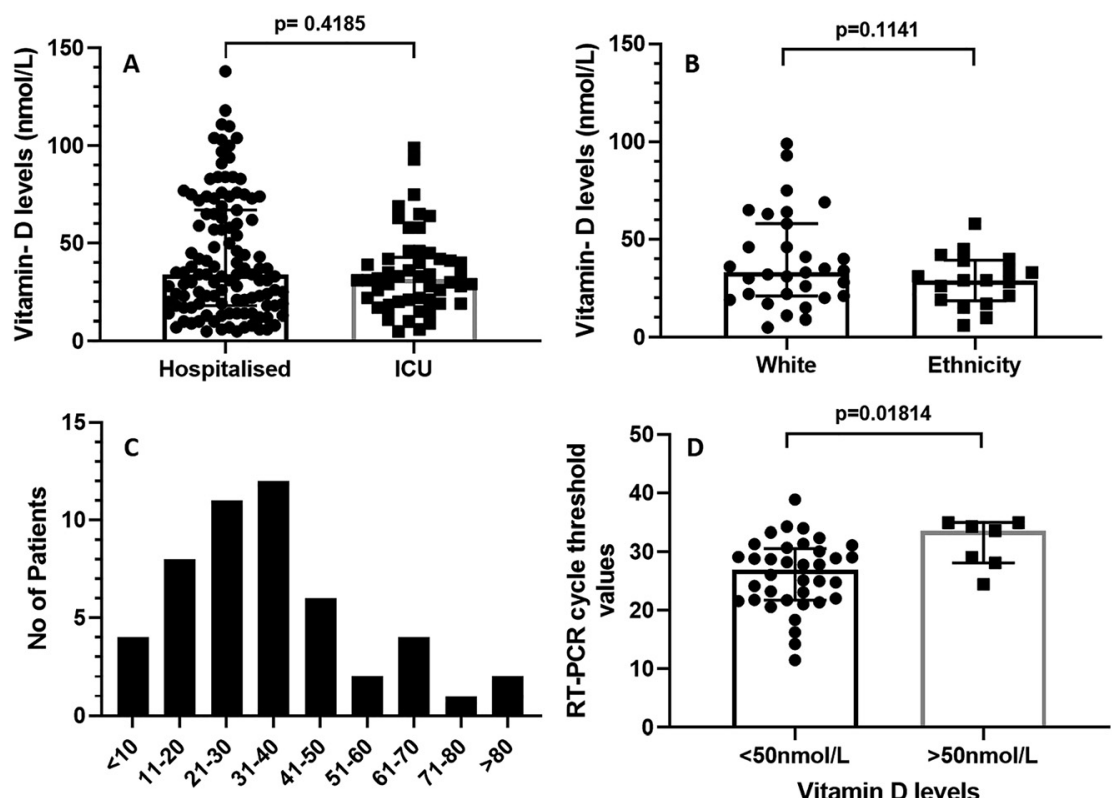

Ranges of Vitamin-D levels (nmol/L)
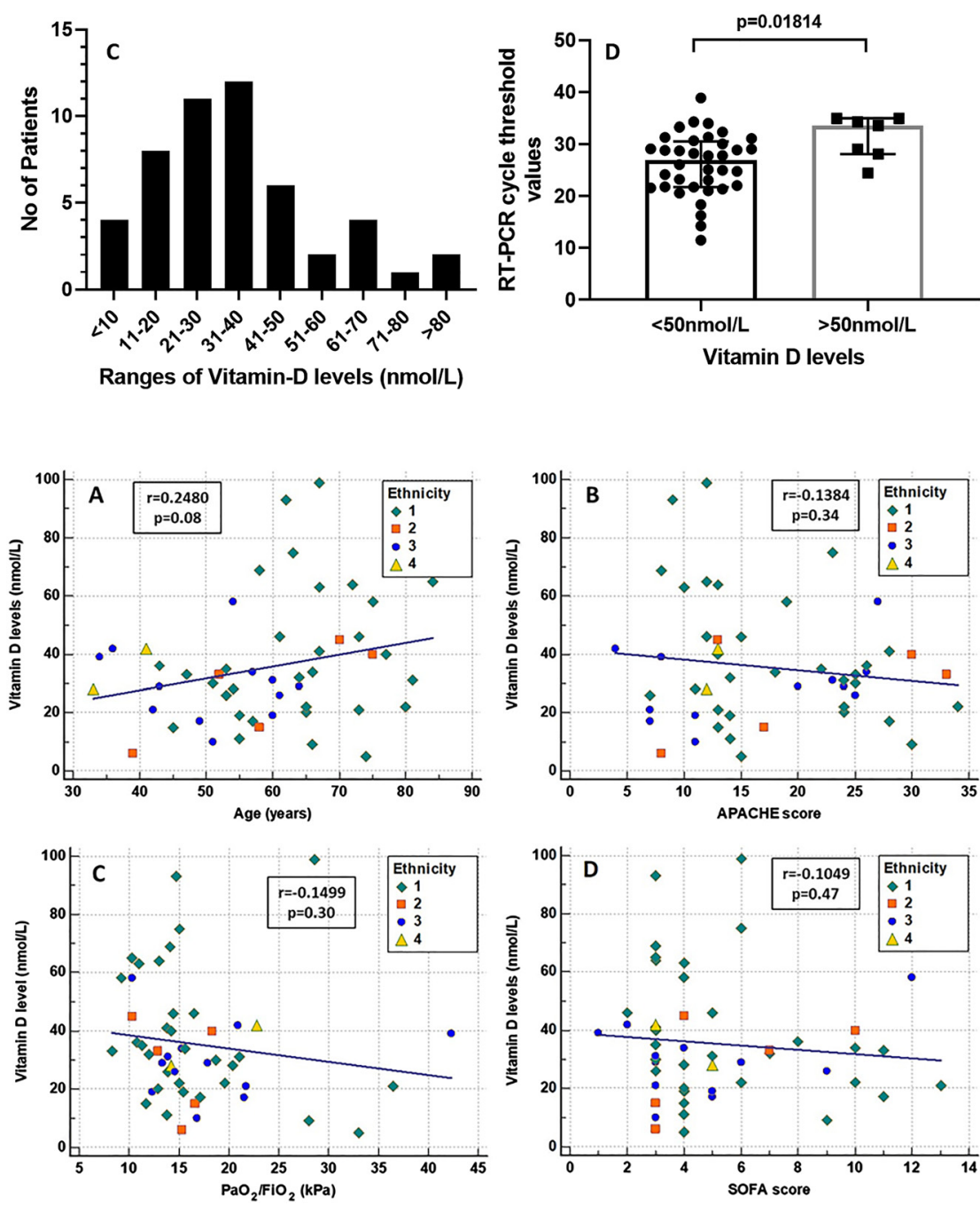

Figure 2: Comparison of vitamin-D levels. (A) Comparison of vitamin-D levels between non-ICU hospitalized and ICU patients.

(B) Vitamin-D levels between white Caucasian and non-white ethnic groups (ethnicity other than white Caucasians). (C) The ranges of vitamin-D levels in the ICU population as presented between $<10$ and $>80 \mathrm{nmol} / \mathrm{L}$. (D) Comparison of RT-PCR values of ICU patients with normal ( $>50 \mathrm{nmol} / \mathrm{L})$ and low ( $<50 \mathrm{nmol} / \mathrm{L})$ vitamin$D$ levels. All boxes in subsets A, B and D show median and interquartile ranges and the dots show individual observations. Comparisons between groups were made with Mann-Whitney $U$ test and a $p$ value of $<0.05$ was considered statistically significant.

Figure 3: Pictorial scatter plots for correlations between vitamin-D levels with age (A), and ICU severity scores Acute Physiology and Chronic Health Evaluation (APACHE II) score (B), admission worst $\mathrm{PaO}_{2} / \mathrm{FiO}_{2}$ ratio $(\mathrm{C})$ and Sequential Organ Failure Assessment (SOFA) Score (D). Each dot represents a patient with varying ethnicity (1. White Caucasian, 2. Black, 3. Asian, 4. Other/mixed or unknown). Correlations were made with Pearson Correlation Coefficient $r$ and $p$-value of $<0.05$ was considered statistical significance.

\section{Discussion}

This observational study suggests that a high proportion (70.3\%) of patients hospitalised with COVID-19 had vitamin-D deficiency. A third of those hospitalised were subsequently admitted to ICU with critical illness, the majority of which required mechanical ventilation. In comparison to those not requiring ICU admission (65.2\%), a greater proportion (82\%) of critically ill COVID-19 patients had vitamin-D deficiency. This is much higher than the anticipated incidence of vitamin-D deficiency in the UK population, which is around $20 \%$ [12].

Within ICU, the group with low vitamin-D ( $<50 \mathrm{nmol} / \mathrm{L})$ levels was significantly younger than the normal group (67 vs. 57 years), and although it is not possible to make direct assumptions, this finding may suggest that younger patients with low vitamin-D are disproportionately affected by COVID-19 for their age. Within this deficient cohort, ethnic patients comprised over $40 \%$, as they were younger than their Caucasian counterparts and had lower vitamin-D levels, 
Table 2: Outcome of ICU patients with vitamin-D levels $>50$ and $<50 \mathrm{ng} / \mathrm{mL}$.

\begin{tabular}{lrrr}
\hline Outcomes & $\begin{array}{r}\text { Vitamin-D level } \\
>\mathbf{5 0} \mathbf{~ n g} \mathbf{m L}, \mathbf{n = 9}\end{array}$ & $\begin{array}{r}\text { Vitamin-D level } \\
<50 \mathbf{~ n g} \mathbf{m L}, \mathbf{n = 4 1}\end{array}$ & p-Value \\
\hline $\begin{array}{l}\text { Length of hospital } \\
\text { stay, days }\end{array}$ & $43.5(34.8)$ & $34(34.0)$ & 0.79 \\
$\begin{array}{l}\text { Invasive mechani- } \\
\text { cal ventilation }\end{array}$ & $6(66.7 \%)$ & $30(73.2 \%)$ & 0.70 \\
$\begin{array}{l}\text { Non-invasive venti- } \\
\text { lation only }\end{array}$ & $2(22.2 \%)$ & $8(19.5 \%)$ & 1.00 \\
$\begin{array}{l}\text { No respiratory } \\
\text { support }\end{array}$ & $1(11.1 \%)$ & $3(7.3 \%)$ & 0.57 \\
$\begin{array}{l}\text { Acute kidney injury } \\
\text { Death }\end{array}$ & $4(44.4 \%)$ & $24(58.5 \%)$ & 0.48 \\
Discharged home & $1(11.1 \%)$ & $6(14.6 \%)$ & 1.00 \\
\hline
\end{tabular}

AKI, acute kidney injury; ICU, intensive care unit; RRT, renal replacement therapy. Data are presented as median (interquartile range) or numbers (percentage) unless otherwise stated.

which may partly explain the inverse relationship between age and vitamin-D deficiency. This finding of young ICU patients with vitamin-D deficiency has also been previously shown by a study of critically ill COVID-19 patients in the UK [13].

Despite this age difference between the groups, there was no association between ICU severity indices (APACHE II and SOFA scores) and vitamin-D levels. Moreover, there were no differences in ICU outcomes (mortality, need for mechanical ventilation and development of acute kidney injury) between the groups. However, the degree of hypoxia as defined by the $\mathrm{PaO}_{2} / \mathrm{FiO}_{2}$ ratio on admission was slightly higher for patients with low vitamin-D levels, which was an unexpected finding compared to previously reported ARDS studies [14].

Vitamin-D deficiency is a common finding in critically ill patients, and lower levels are associated with adverse outcomes $[15,16]$. In contrast, our study did not show any differences in clinical outcomes and vitamin-D levels. This may be due to several reasons: (1) our study consisted of small sample size, preventing any robust correlations or clinical extrapolations; (2) there may be other variables such as patient-specific risk factors, ICU interventions and bacterial co-infections, that may influence the overall clinical outcomes more than vitamin-D levels alone.

For the critically ill ICU patients, we calculated the cycle threshold (CT) for polymerase chain reaction (PCR) used to detect the viral RNA. The CT value represents the amount of replication cycles required during real time PCR to expand the target sample up over a threshold level. A higher initial sample concentration of a target gene will require fewer cycles to achieve threshold level, therefore a lower CT value [17]. CT value can therefore be used as an indirect measure of viral load. However, this must be viewed with caution, as the PCR is not fully quantitative and CT values may vary from one sample to another. Interestingly we showed that the ICU group with low vitamin-D levels ( $<50 \mathrm{nmol} / \mathrm{L}$ ) had a significantly lower mean CT value ( 27.0 vs. $33.6, \mathrm{p}=0.02$ ) compared to the patients with normal vitamin-D ( $>50 \mathrm{nmol} / \mathrm{L})$. To our knowledge, this finding has not been published previously. Lower CT values have been linked to increased severity and increased mortality from COVID-19, although this link remains disputed [17-19].
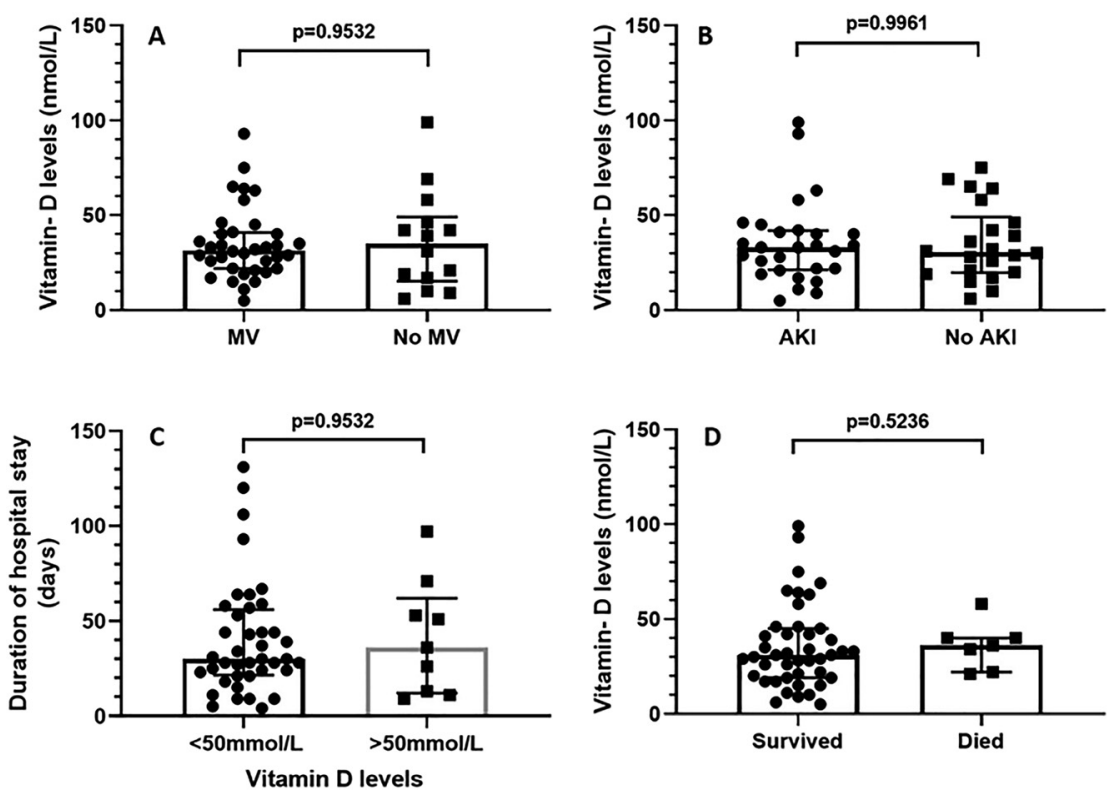

Figure 4: ICU outcome variables and vitamin-D levels.

(A) The need for mechanical ventilation (MV). (B) The development of acute kidney injury (AKI). (C) The hospital length of stay (days). (D) Survival outcome. All boxes show median and interquartile ranges, and the dots show individual observations. Comparisons between groups were made with Mann-Whitney $U$ test and a $p$ value of $<0.05$ was considered statistically significant. 
SARS-CoV- 2 is an RNA stranded BETA coronavirus and enters the host primarily by via the respiratory tract. The surface glycoprotein (Spike protein) binds to the host cells via the angiotensin converting enzyme 2 (ACE2), which is highly expressed on the alveolar epithelial cells. It appears that the severity of the disease is dependent on the host's innate susceptibility to infection, and the extent of the host's inflammatory response once infected. In severe cases, local inflammation in the lungs leads to a local lung injury, similar to Acute Respiratory Distress Syndrome (ARDS) with local cytokine production (particularly IL-6 and IL-8) driving the infiltration of large numbers of immune cells, including neutrophils and T-cells. This may be combined with a systemic 'cytokine storm' driven by T-helper cells leading to multi-organ derangement requiring intensive support $[1,3]$. It is thought that T-regulatory cells have an important role in moderating this systemic inflammatory response, curtailing the runaway positive feedback of a cytokine storm. There are several potential underlying mechanisms which may explain the association between vitamin-D deficiency and COVID-19 susceptibility and severity [20].

Apart from the commonly known function on bone health, vitamin-D appears to have a role in immune modulation and control via the vitamin-D receptor (VDR). It has been demonstrated that vitamin-D up-regulates the expression of ACE2 [21], this could help to counter the downregulation caused by the binding of SARS-CoV-2, and therefore, help protect against the pro-inflammatory and vasoconstrictive effects of a build-up of angiotensin-II (1-8) [22]. This has been demonstrated in mouse models, where pre-treatment with vitamin-D was protective against ARDS [21]. Vitamin-D has been shown to influence both innate and adaptive immunity during infections [23]. It modifies macrophage and monocyte activity, reducing the circulating levels of pro-inflammatory cytokines [24-26]. Vitamin-D has been also shown to influence human adaptive immune response of T-cells, promoting a humoral Th2 as opposed to the Th1, which is also linked to pro-inflammatory cytokine release [27]. Moreover, it has direct antiviral properties, particularly against enveloped respiratory viruses [28].

The association of low vitamin-D levels and clinical COVID-19 infection needs further exploration. Large studies of historical databases have shown that people with low vitamin-D levels are more likely to become infected with the SARS-COV-2 virus [29, 30]. Moreover, there is a temporal link between low vitamin-D levels and both risk of infection and hospitalization [31]. However, contrary to these findings, UK studies using historical 'BIOBANK' data, show that, corrected for multivariant factors, there is no significant link between COVID-19 and
vitamin-D deficiency [32-34]. However, these are large retrospective studies which rely on historical vitamin-D levels from volunteers taken over 10 years ago and may not be reflective of the current nutritional status during the COVID-19 pandemic. Moreover, although there is some evidence that vitamin-D status will remain consistent, it is likely that there will be large enough variation over this timescale to making these results difficult to interpret, particularly in those hospitalised and with critical illness. Another recent study from the UK used a prospective cohort model with 103 patients presenting with symptoms of COVID-19. Vitamin-D levels were tested on admission, and of these patients, 70 were positive for SARS-COV-2. This positive group had a significantly lower vitamin-D level $(27 \mathrm{nmol} / \mathrm{L})$ compared to the non-positive group (52 nmol/L) [35]. Although patients with lower vitamin-D levels ( $<30 \mathrm{nmol} / \mathrm{L}$ ) had increased D-Dimer, non-invasive ventilation requirements and critical care admissions, there were no differences in the mortality [35].

Despite this growing evidence, we found no association of vitamin-D deficiency and ICU severity indices and outcomes. Studies focused on COVID-19 and vitamin-D measurements specific to ICU population are lacking. Similar to our findings, using $50 \mathrm{nmol} / \mathrm{L}$ as the cut off value, a retrospective study of ICU patients showed a much higher rate of vitamin-D deficiency among the ICU patients compared to non-ICU patients [13]. Despite this association, there is no outcome difference. There may be several reasons for these findings. Firstly, our cohort consists of a specific critically ill patient group and their outcome is likely influenced by a number of factors, rather than a single nutritional variable. Secondly, we used the cut off for the normal values as $50 \mathrm{nmol} / \mathrm{L}$ as guided by the local protocols. However, we did not see any direct correlation between the degree of vitamin-D deficiency and ICU outcomes. Thirdly, our study consisted of a small sample size, particularly in the normal group $(n=9)$ and may not have the statistical power to detect a robust clinical outcome. However, it is reassuring that despite a high prevalence of vitamin-D deficiency with associated reduced viral CT-PCR status, the intensive care outcomes are relatively comparable between patients with normal and low values.

\section{Conclusions}

In our observational study, ICU admission with COVID-19 was associated with a higher incidence of vitamin-D deficiency. This is unexpected given the lower age of this cohort. Critically ill COVID-19 patients with low vitamin-D levels were found to be younger with lower CT-PCR levels, 
which could imply a higher viral load. There were no measurable differences in overall clinical outcomes between those with normal $(>50 \mathrm{nmol} / \mathrm{L})$ and deficient $(<50 \mathrm{nmol} / \mathrm{L})$ vitamin-D levels. However, this was a small observational study specific to critically ill COVID-19 patients, so the possibility for detailed analysis or clinical inferences is more limited. If vitamin-D influences the susceptibility or severity of COVID-19, it is likely via a complex immune modulatory process, and this may be difficult to measure in uncontrolled observational studies such as this one. There is a clear need for large randomized controlled trials to correctly measure the effect, if any, that vitamin-D has on COVID 19.

Research funding: None declared.

Author contributions: All authors have accepted responsibility for the entire content of this manuscript and approved its submission.

Competing interests: Authors state no conflict of interest. Informed consent: Informed consent was obtained from all individuals included in this study.

Ethical approval: This study was part of a larger observational cohort study (REACT-COVID Observational Database) and has the approval of the local research and development and National Heath Research Authority (IRAS:285145) with a REC Reference 20/HRA/2986.

\section{References}

1. Tabary M, Khanmohammadi S, Araghi F, Dadkhahfar S, Tavangar S. Pathologic features of COVID-19: a concise review. Pathol Res Pract 2020;216:153097.

2. Group TRC. Dexamethasone in hospitalized patients with Covid19 - preliminary report. N Engl J Med 2020 Jul 17. https://doi.org/ 10.1056/NEJMoa2021436 [Epub ahead of print].

3. Yuki K, Fujiogi M, Koutsogiannaki S. COVID-19 pathophysiology: a review. Clin Immunol 2020;215:108427.

4. Chandran M, Maung AC, Mithal A, Parameswaran R. Vitamin D in COVID-19: dousing the fire or averting the storm? - a perspective from the Asia-Pacific. Osteoporos Sarcopenia 2020 Jul 23. https:// doi.org/10.1016/j.afos.2020.07.003 [Epub ahead of print].

5. Im JH, Je YS, Baek J, Chung MH, Kwon HY, Lee JS. Nutritional status of patients with coronavirus disease 2019 (COVID-19). Int J Infect Dis 2020 Aug 11. https://doi.org/10.1016/j.ijid.2020.08.018 [Epub ahead of print].

6. Mitchell F. Vitamin-D and COVID-19: do deficient risk a poorer outcome? Lancet Diabetes Endocrinol 2020;8:570.

7. Weir EK, Thenappan T, Bhargava M, Chen Y. Does vitamin D deficiency increase the severity of COVID-19? Clin Med 2020;20: e107-8.

8. Pham H, Rahman A, Majidi A, Waterhouse M, Neale RE. Acute respiratory tract infection and 25 -hydroxyvitamin $D$ concentration: a systematic review and meta-analysis. Int J Environ Res Publ Health 2019;16:3020.
9. Lofgren E, Fefferman NH, Naumov YN, Gorski J, Naumova EN. Influenza seasonality: underlying causes and modeling theories. J Virol 2007;81:5429-36.

10. NICE. COVID-19 rapid evidence summary: vitamin D for COVID-19 advice NICE. Available from: https://www.nice.org.uk/advice/ es28/chapter/Key-messages [Accessed 7 Sep 2020].

11. Kellum JA, Lameire N, Aspelin P, Barsoum RS, Burdmann EA, Goldstein SL, et al. Kidney disease: improving global outcomes (KDIGO) acute kidney injury work group. KDIGO clinical practice guideline for acute kidney injury. Kidney Int Suppl 2012;2: 1-138.

12. Bates B, Lennox A, Prentice A, Bates C, Swan G. National diet and nutrition survey; 2011. Available from: https://assets.publishing. service.gov.uk/government/uploads/system/uploads/ attachment_data/file/207708/NDNS-Y3-report_All-TEXT-docscombined.pdf.

13. Panagiotou G, Tee SA, Ihsan Y, Athar W, Marchitelli G, Kelly D, et al. Low serum 25-hydroxyvitamin D (25[OH]D) levels in patients hospitalised with COVID-19 are associated with greater disease severity. Clin Endocrinol 2020 Jul 3. https://doi.org/10.1111/cen. 14276 [Epub ahead of print].

14. Dancer RCA, Parekh D, Lax S, D'Souza V, Zheng S, Bassford CR, et al. Vitamin $D$ deficiency contributes directly to the acute respiratory distress syndrome (ARDS). Thorax 2015;70:617-24.

15. Rech MA, Hunsaker T, Rodriguez J. Deficiency in 25-hydroxyvitamin $D$ and 30-day mortality in patients with severe sepsis and septic shock. Am J Crit Care 2014;23:e72-9.

16. Anwar E, Hamdy G, Taher E, Fawzy E, Abdulattif S, Attia MH. Burden and outcome of vitamin D deficiency among critically ill patients: a prospective study. Nutr Clin Pract 2017;32:378-84.

17. Rao SN, Manissero D, Steele VR, Pareja J. A narrative systematic review of the clinical utility of cycle threshold values in the context of COVID-19. Infect Dis Ther 2020;9:1-14.

18. Huang J-T, Ran R-X, Lv Z-H, Feng LN, Ran CY, Tong YQ, et al. Chronological changes of viral shedding in adult inpatients with COVID-19 in Wuhan, China. Clin Infect Dis 2020 May 23. https:// doi.org/10.1093/cid/ciaa631 [Epub ahead of print].

19. Yu F, Yan L, Wang N, Yang S, Wang L, Tang Y, et al. Quantitative detection and viral load analysis of SARS-CoV-2 in infected patients. Clin Infect Dis 2020 Mar 28. https://doi.org/10.1093/ cid/ciaa345 [Epub ahead of print].

20. Aygun H. Vitamin D can prevent COVID-19 infection-induced multiple organ damage. Naunyn-Schmiedeberg's Arch Pharmacol 2020;393:1157-60.

21. Xu J, Yang J, Chen J, Luo Q, Zhang Q, Zhang H. Vitamin D alleviates lipopolysaccharide-induced acute lung injury via regulation of the renin-angiotensin system. Mol Med Rep 2017;16:7432.

22. Quesada-Gomez JM, Entrenas-Castillo M, Bouillon R. Vitamin D receptor stimulation to reduce acute respiratory distress syndrome (ARDS) in patients with coronavirus SARS-CoV-2 infections: revised Ms SBMB 2020_166. J Steroid Biochem Mol Biol 2020;202:105719.

23. Charoenngam N, Holick MF. Immunologic effects of vitamin D on human health and disease. Nutrients 2020 Jul 15;12. https://doi. org/10.3390/nu12072097 [Epub ahead of print].

24. Adams JS, Ren S, Liu PT, Chun RF, Lagishetty V, Gombart AF, et al. Vitamin D-directed rheostatic regulation of monocyte antibacterial responses. J Immunol 2009;182:4289-95.

25. Almerighi C, Sinistro A, Cavazza A, Ciaprini C, Rocchi G, Bergamini A, et al. 1alpha,25-dihydroxyvitamin D3 inhibits 
CD40L-induced pro-inflammatory and immunomodulatory activity in human monocytes. Cytokine 2009;45:190-7.

26. Zhang Y, Leung DYM, Richers BN, Liu Y, Remigio LK, Riches DW, et al. Vitamin D inhibits monocyte/macrophage proinflammatory cytokine production by targeting MAPK phosphatase-1. J Immunol 2012;188:2127-35.

27. Di Rosa M, Malaguarnera M, Nicoletti F, Malaguarnera L. Vitamin D3: a helpful immuno-modulator. Immunology 2011; 134:123-39.

28. Barlow PG, Svoboda P, Mackellar A, Nash AA, York I, Pohl J, et al. Antiviral activity and increased host defense against influenza infection elicited by the human cathelicidin LL-37. PloS One 2011; 6:e25333.

29. Meltzer DO, Best TJ, Zhang H, Vokes T, Arora V, Solway J. Association of vitamin $\mathrm{D}$ deficiency and treatment with COVID-19 incidence. medRxiv 2020 May 13. https://doi.org/10.1101/2020. 05.08.20095893 [Epub ahead of print].

30. D’Avolio A, Avataneo V, Manca A, Cusato J, De Nicolò A, Lucchini R, et al. 25-hhydroxyvitamin $D$ concentrations are lower in patients with positive PCR for SARS-CoV-2. Nutrients 2020 May 9;12. https://doi.org/10.3390/nu12051359 [Epub ahead of print].

31. Merzon E, Tworowski D, Gorohovski A, Vinker S, Cohen AG, Green I, et al. Low plasma $25(\mathrm{OH})$ vitamin D level is associated with increased risk of COVID-19 infection: an Israeli populationbased study. FEBS I 2020 Jul 23. https://doi.org/10.1111/febs. 15495 [Epub ahead of print].

32. Hastie CE, Mackay DF, Ho F, Celis-Morales CA, Katikireddi SV, Niedzwiedz CL, et al. Vitamin D concentrations and COVID-19 infection in UK Biobank. Diabetes Metab Syndr 2020;14:561-5.

33. Hastie CE, Pell JP, Sattar N. Vitamin D and COVID-19 infection and mortality in UK Biobank. Eur J Nutr 2020 Aug 26. https://doi.org/ 10.1007/s00394-020-02372-4 [Epub ahead of print].

34. Raisi-Estabragh Z, McCracken C, Bethell MS, Cooper J, Cooper C, Caulfield MJ, et al. Greater risk of severe COVID-19 in Black, Asian and Minority Ethnic populations is not explained by cardiometabolic, socioeconomic or behavioural factors, or by 25(OH)-vitamin D status: study of 1,326 cases from the UK Biobank. J Public Health 2020 Jun 19. https://doi.org/10.1093/ pubmed/fdaa095 [Epub ahead of print].

35. Baktash V, Hosack T, Patel N, Shah S, Kandiah P, Van Den Abbeele K, et al. Vitamin $D$ status and outcomes for hospitalised older patients with COVID-19. Postgrad Med J 2020 Aug 27. https://doi.org/10.1136/ postgradmedj-2020-138712 [Epub ahead of print].

Supplementary Material: The online version of this article offers supplementary material (https://doi.org/10.1515/cclm-2020-1567). 